\title{
Las plataformas de materiales didácticos digitales en Educación Infantil: ¿están adaptadas a las características del alumnado?
}

\author{
The platforms of digital teaching materials in Early Childhood \\ Education: are they adapted to students' characteristics?
}

RECIBIDO 30/3/2021 ACEPTADO 31/5/2021 PUBLICADO 1/12/2021

\section{Rebeca Fernández Iglesias}

Facultad de Educación, Universidade de Santiago de Compostela, España

rebeca.fernandez@rai.usc.es

\section{(D) María del Camino Pereiro González}

Xunta de Galicia, Consellería de Educación, Cultura e Universidade, CPI Plurilingüe Virxe da Cela (Monfero), España

pereirogonzalez@edu.xunta.gal

\section{(iD) Antía Cores Torres}

Facultad de Educación, Universidade de Santiago de Compostela, España

antia.cores.torres@usc.es

(iD) Daniela Gonçalves

ESE Paula Frassinetti. CIDTFF da Universidade de Aveiro, CIPAF da ESEPF, Portugal

dag@esepf.pt

\section{RESUMEN}

En los últimos años ha aumentado la presencia de la tecnología en las aulas de nuestros centros educativos, y la etapa de Educación Infantil no ha quedado al margen de este acontecimiento. Esta realidad despertó interés para desarrollar el proyecto de I+D+i "Los materiales didácticos digitales en la Educación Infantil. Análisis y propuestas para su uso en la escuela y el hogar. Infanci@ Digit@l” en el que se enmarca este artículo. El objetivo del mismo es realizar una comparativa entre cinco plataformas de materiales didácticos digitales de diversa naturaleza (institucionales, editoriales y de acceso libre) de la comunidad autónoma gallega. Para ello, se aplica una guía de evaluación que se centra en las características tecnológicas, pedagógicas y socio-comunicacionales de dichas plataformas. Del mismo modo, se tienen en cuenta si estos recursos atienden a las características, necesidades e intereses del alumnado de la etapa educativa a la que van destinados. A través de este análisis llegamos a la conclusión de que las plataformas se están adaptando a la realidad de nuestra sociedad, pero de manera muy paulatina y superficial.

PALABRAS CLAVE Educación Infantil, Plataformas Digitales, Recursos Educativos.

\section{ABSTRACT}

In recent years, the presence of technology in the classrooms of our educational centers has increased and the Early Childhood Educational stage has not been left out of this event. This reality aroused interest to develop the I+D+I project "Digital 
Didactic Materials for Early Childhood Education. Analysis and proposals for use at school and at home. Digit@l Childhood" in which this article is framed. The objective of this work is to make a comparison between five platforms of digital didactic materials of diverse natures (institutional, editorial and free access) of Galicia. For this, we applied an evaluation guide that focuses on the technological, pedagogical, and socio-communicational characteristics of these platforms. In the same way, it is taken into account whether these resources know the characteristics, needs and interests of the students of this educational stage to which they are intended. Throw this analysis we conclude that the platforms are adapting them to the reality of our society, but in a very gradual and superficial way.

KEYWORDS Early Childhood Education, Digital Platforms, Educational Resources.

\section{INTRODUCCIÓN}

La introducción de cambios, vinculados al acto de aprender y enseñar a la luz de un nuevo paradigma didáctico-pedagógico e impulsado por nuevos conceptos de educación, nuevas habilidades, atributos y capacidades, ha sido justificada por las profundas transformaciones que atraviesa la sociedad actual, que requieren el reajuste del papel de la escuela y la reestructuración de los procesos de enseñanza y aprendizaje.

Esta nueva forma de entender la práctica se basa en una actitud de cuestionamiento (Gonçalves, 2015), sustentada en referencias teóricas de análisis, en el dominio de metodologías adecuadas y, claramente, en el afán de conocer mejor y actuar mejor. Influye también, en esta adaptación, el cambio tan obvio que observamos en las experiencias de la infancia actual (Chaudron et al., 2018).

La sociedad contemporánea, marcada por rápidos cambios sociales, educativos, psicológicos, tecnológicos, entre otros, se denomina desde hace algunos años Sociedad de la Información. Algunos autores (Flórez et al., 2017; Hernández, 2017; Pérez et al., 2018) optan por el nombre Sociedad del Conocimiento. Más que estas denominaciones, lo cierto es que estamos claramente en una sociedad marcada por lo digital. Actualmente, en todos los aspectos de la vida profesional o incluso en el ámbito personal, es casi imposible no tener contacto con las tecnologías digitales e Internet.

Algunos estudios (Gottskchalk, 2019) muestran que no hay evidencia de que los jóvenes sean, de hecho, competentes digitalmente. Existe, por tanto, la necesidad de desarrollar itinerarios formativos adecuados para todos, incluidos los más jóvenes, porque, de hecho, ser un "nativo digital”, en sí mismo, no confiere competencias. Cada persona debe avanzar hacia la adquisición de una inteligencia digital no innata, una especie de sabiduría digital según Gutiérrez (2018), y que puede ser la base de la ciudadanía plena.

Existe una tendencia positiva, pero no absoluta, de que el uso de tecnologías y entornos interactivos (digitales), incluidos los recursos digitales, favorecen el aprendizaje y su calidad. Se menciona, de manera sostenida, que existe una mayor implicación y motivación de los actores, es decir, estudiantes y profesores (Hooft, 2018). Por tanto, las creencias educativas, los conocimientos y las habilidades de los actores juegan aquí un papel destacado.

Además, se pone especial interés en el fomento del aprendizaje activo en el que el alumno pasa de ser un sujeto pasivo en el proceso de enseñanza-aprendizaje a ser un elemento activo. Este aprendizaje no solo desarrolla habilidades sociales, sino que propicia el que sea más profundo y permanente, contribuyendo a 
la generación de mayores posibilidades de éxito (Altun, 2017), al considerar el logro individual dependiente del logro del grupo que se ayuda mutuamente a lo largo del proceso.

Lo que está claro es que la combinación de ambos retos (la integración de las TIC en las aulas y la necesaria innovación didáctica que ello requiere) supone una transformación digital de las organizaciones educativas, entendido este hecho como un proceso complejo, disruptivo y en el que se entrecruzan factores de distinta naturaleza (tecnológicos, organizativos y culturales) (Sosa, \& Valverde, 2020).

En este sentido, durante los últimos años ha aumentado notablemente la producción de materiales didácticos digitales, destinados para niños y niñas de Educación Infantil, al mismo tiempo que se impulsó su uso en las aulas y en los hogares. Este incremento en la producción y utilización también ha generado la necesidad de organizar, categorizar y sistematizar los innumerables recursos que se encuentran en la red, y es así como surgen las plataformas educativas. Estos portales en la web que congregan en un mismo sitio innumerables recursos son definidos por González et al. (2018) como:

Sitios web que se ponen a disposición de la comunidad educativa, ofertando servicios y contenidos que potencien el proceso de enseñanza-aprendizaje desde un entorno más llamativo para el alumnado (...) están destinadas a un uso didáctico, por ello, recopilan recursos educativos de todo tipo: interactivos, audiovisuales, textuales... (p. 90)

Gabarda et al. (2021), tras la revisión de investigaciones previas de diferente autoría, sistematizan las 3 principales premisas que cumplen estas plataformas de materiales didácticos digitales:

- Tienen una función informativa al ofrecer datos de interés para los destinatarios y formativa al incluir recursos orientados a la mejora de las competencias de los usuarios.

- Pueden considerarse espacios de almacenamiento de información y documentación: materiales didácticos, noticias, espacios de colaboración, etc.

- Son colaborativos, siendo la clave que los docentes compartan recursos que elaboran de manera individual o colectiva.

En cuanto a su clasificación, Area (2003) propone una división de estas plataformas en cuatro tipos fundamentales: webs institucionales, webs de recursos y bases de datos educativos, entornos de teleformación e intranets educativas y materiales didácticos web, que cumplen finalidades diferentes. De este modo, en los dos primeros prevalece la información mientras que en los dos últimos es más importante la formación. Teniendo en consideración esta taxonomía, en el proyecto de investigación del que emana esta publicación se propone una clasificación de las plataformas en tres tipos en función del organismo, institución o empresa privada que las sustentan:

- Institucionales: gestionadas por las Consejerías de Educación y por el Ministerio de Educación a través del portal Procomún Educalab del INTEF (Instituto Nacional de Tecnologías y de la Información y Formación del profesorado).

- Comerciales: sustentadas por empresas privadas que producen contenidos educativos para Educación Infantil, como Anaya, Bruño, Xerais, etc.

- De acceso libre: impulsadas por diferentes organismos, como bibliotecas, museos, cooperativas, etc. 


\section{MATERIAL Y MÉTODO}

Esta investigación se sitúa en el marco del proyecto de investigación titulado: "Los materiales didácticos digitales en la Educación Infantil. Análisis y propuestas para su uso en la escuela y el hogar”, cuyo objetivo general consiste en "explorar y analizar las características técnicas y pedagógicas de los MDD dirigidos al alumnado del segundo ciclo de Educación Infantil, así como los usos y prácticas desarrollados con los mismos en el contexto escolar y familiar" (Rodríguez, \& Area, 2019).

El proyecto plantea un total de cuatro estudios diferentes y en esta publicación nos centramos en el Estudio I, cuyo objetivo general es analizar las características pedagógicas y tecnológicas de una muestra de repositorios y plataformas de materiales educativos digitales actualmente existentes en España destinadas al segundo ciclo de Educación Infantil tanto de plataformas de contenidos educativos comerciales como de repositorios institucionales públicos.

De un modo más específico, este Estudio I pretende responder a los siguientes objetivos:

1. Realizar un análisis de una muestra de plataformas comerciales de materiales y recursos digitales destinados a niños y niñas de 3 a 6 años.

2. Realizar un análisis de los repositorios institucionales creados por las Consejerías de Educación en Canarias, Galicia y Valencia.

3. Realizar un análisis comparativo entre plataformas digitales analizadas y una muestra de distintos tipos de recursos (videojuegos, apps, juegos electrónicos, etc.).

Desde el punto de vista metodológico, en este artículo se opta por un enfoque cualitativo basado en el análisis de contenido. Para realizar este análisis de las plataformas de materiales didácticos digitales de Educación Infantil, se utiliza una guía de evaluación. Este instrumento de análisis de portales web se ha adaptado de un instrumento previo utilizado en un proyecto anterior (Escuel@ Digit@l), validado por expertos en Tecnología Educativa y Didáctica y Organización Escolar.

La muestra está constituida por plataformas de materiales didácticos digitales, destinados a niños y niñas entre 3 y 6 años y albergadas en tres Comunidades Autónomas: Galicia, Canarias y Comunidad Valenciana. No obstante, en este artículo se aborda el análisis de una pequeña parte de la muestra total conformada por cinco plataformas de la Comunidad Autónoma gallega cuyas principales características se resumen en la siguiente tabla:

TABLA 1. Distribución de la muestra

\begin{tabular}{lll}
\hline & TIPO & ENLACE \\
\hline Kalandraka & Comercial & $\underline{\text { http://www.kalandraka.com/ga/ }}$ \\
\hline Bruño & Comercial & $\underline{\text { https://www.editorial-bruno.es/digitalinfantil.php }}$ \\
\hline Alíalí-Fabaloba & Acceso libre & $\underline{\text { http://aliali.fabaloba.com/ }}$ \\
\hline Orella Pendella & Acceso libre & $\underline{\text { https://orellapendella.gal// }}$ \\
\hline EspazoAbalar & Institucional & $\underline{\text { https://www.edu.xunta.gal/espazoAbalar/ }}$ \\
\hline Total & $\mathbf{5}$ & \\
\hline
\end{tabular}


El instrumento de recogida de información, la guía de evaluación, ha sido elaborado por el equipo de investigación y validado por expertos en dicha temática. En cuanto a su estructura, se divide en las siguientes 5 dimensiones:

1. Datos de identificación del portal o plataforma web: título, empresa o institución, dirección web, idiomas utilizados, naturaleza del portal y tipo de acceso.

2. Estructura de la plataforma: descripción general de la página inicial haciendo hincapié en su organización (secciones, menú y submenús), en su diseño e interfaz gráfica y en la plataforma tecnológica que soporta el portal (tablet, ordenador, smartphone).

3. Recursos educativos disponibles en el portal: se clasifica la tipología de recursos disponibles en el portal (objetos digitales, objetos digitales de aprendizaje, materiales didácticos digitales, materiales profesionales para docentes y aplicaciones o herramientas informáticas).

4. Servicios y entornos para la gestión de la información y comunicación: identificación y descripción de los servicios ofertados (foros, noticias, redes sociales, calendarios, enlaces, videoconferencias, etc.) y su importancia en la plataforma

5. Valoración global de la plataforma destacando las características tecnológicas, pedagógicas y sociocomunicacionales y valorando sus fortalezas y debilidades.

En el siguiente apartado se recogerán, principalmente, los resultados obtenidos en este último punto de la guía de evaluación utilizada para el análisis de las plataformas de materiales didácticos digitales para Educación Infantil.

\section{RESULTADOS}

Se analizaron, tal y como comentamos, cinco plataformas a nivel autonómico: una de la administración pública, dos comerciales propias de editoriales y los dos restantes libres, sin relación directa con ninguna firma comercial o institucional.

En líneas generales, destacan en su diseño por atender a las finalidades propias de su sustento fundacional.

El “EspazoAbalar”, plataforma de la administración educativa gallega muestra un marcado carácter institucional e informativo agrupando diversos servicios para la comunidad educativa, cumpliendo con los estándares de accesibilidad básicos (Gonzales, 2016), pero con ciertas limitaciones en sus funcionalidades. Aparenta contar con gran número de posibilidades para el colectivo destinatario, pero, al final las mismas son reducidas, re-enlazadas y escasamente actualizadas. Así, a nivel tecnológico, su diseño y desarrollo muestra una línea corporativa bien definida, pero sin evolución temporal.

En el ámbito editorial, por un lado, está la propuesta de Kalandraka que, sobre todo, parece estar diseñada como recurso divulgativo de su catálogo de obras impresas (de lectura, álbumes ilustrados y bibliografía pedagógica) y de sus actividades comunicativas (ofertas y novedades, actos, premios, recomendaciones literarias y puntos de venta). Su acceso es en abierto, aunque no ofrece la posibilidad de interacción entre usuarios, ni herramientas para publicar en red. Su diseño es sencillo y organizado, aunque con carencias en su accesibilidad si atendemos a los estándares específicos. 
En cuanto a Bruño, ofrece una multiplataforma con acceso a través de registro como persona cliente de la editorial. Su estética es agradable, de fácil manejo, intuitiva y funcional en gran variedad de soportes tecnológicos. Como aspecto diferencial, destacan sus herramientas de gestión, de aula y de centro con posibilidades comunicativas entre el profesorado apoyadas por soporte técnico.

Como propuestas de acceso libre contamos con Aliali-Fabaloba, con unos estándares básicos a nivel tecnológico, pero que destaca en sus redes de comunicación, interacción e intercambio de ideas que, además, son un buen medio para visibilizar y difundir espacios y materiales educativos.

Orellapendella es una plataforma en abierto, sencilla y de fácil navegabilidad que parte del trabajo colaborativo. En ella, priman los aspectos culturales derivados de una comunidad educativa que los difunde y los mantienen vivos en el tiempo y en espacio.

Centrándonos en la vertiente pedagógica, también observamos diversidad de planteamientos, muy relacionados con las finalidades de las propias plataformas.

"EspazoAbalar" no presenta una línea clara, son de gran variedad los recursos ofertados, aunque en ellos priman las actividades "tradicionales" de los libros de texto adaptadas al entorno tecnológico. En demasiadas ocasiones, con poca calidad, sobre todo las de mayor antigüedad. Las más recientes presentan destacadas mejoras, tanto a nivel de accesibilidad como de valores pedagógicos (fundamentalmente los realizados a partir de las licencias por estudios ofertadas desde la administración educativa).

Su compleja accesibilidad dificulta el acceso directo por parte del alumnado, menos aún si nos referimos a la primera infancia. Al mismo tiempo, la divulgación de los materiales prima sobre los planteamientos pedagógicos que se supeditan a la autonomía y profesionalidad del profesorado cuando los implementa.

En el ámbito editorial, Kalandraka centra su oferta digital en su canal de vídeos (Kalandraka TV) con propuestas - grabaciones- adaptadas a un público infantil pero que no permiten su interacción. Así, estaríamos ante materiales que pueden emplearse como recursos didácticos por el profesorado pero que no tienen una clara finalidad pedagógica.

Bruño cuenta con dos tipos de recursos: los materiales didácticos digitales -MDD- y los libros de texto digitales - LTD_, ambos definidos dentro de la Guía para la producción y uso de los materiales didácticos digitales (Area, 2019). Responden a modelos educativos tradicionales enriquecidos con algunas actividades interactivas que no ofrecen la posibilidad de edición por parte del profesorado lo que dificulta las posibilidades de adaptación a las características del alumnado de Educación Infantil, a su contexto y sus diferentes vías de acceso al currículo. La mayoría están editados en lengua castellana, existiendo algunas versiones en catalán, pero ninguna en lengua gallega.

En cuanto a su diseño curricular, prioriza la lectoescritura y el cálculo teniendo poco peso valores, actitudes y conocimientos relacionados con los ámbitos de desarrollo personal y social, que únicamente se presentan en los materiales de proyectos, partiendo los mismos de centros de interés tradicionales de tipo genérico sin posibilidades de adaptación.

Lo que sí ofrece son buenas posibilidades para el desarrollo grafomotor, a través del encerado digital o la tablet excluyendo eso sí, las simulaciones de lo natural. Así, estaríamos ante materiales principalmente disciplinares centrados en el trabajo individual que aumentan de forma lineal su complejidad atendiendo a la dimensión del aprendizaje que se aborde (cálculo, vocabulario, grafía, entre otros). 
Dentro de las plataformas de acceso libre, Aliali-Fabaloba apuesta por un gran banco de recursos agrupados por temáticas entre los que se aprecian criterios propios de los ámbitos de desarrollo de las áreas curriculares de la etapa. Destacan por contar con breves y claras instrucciones de uso, propias para guiar un uso adulto con finalidades educativas. De esta forma, son pocas las actividades destinadas a ser empleadas de forma directa e interactiva por parte de niños y niñas a las que se dirigen. También, se aprecia su pluralidad, al reflejar diversos contextos, realidades y diversidades sociales y culturales de forma que cualquier persona usuaria se puede ver reflejada en alguno de ellos.

Orellapendella sigue la misma base, destacando los contenidos relacionados con las vivencias infantiles, la transmisión de los valores tradicionales e identitarios de la cultura y del saber popular gallego. Son propuestas genéricas sin posibilidades de interacción que se pueden emplear o adaptar por el profesorado a los aspectos curriculares más formales, pero que no resulta atractiva para un uso directo por los niños y niñas de la etapa infantil. De esta forma, destaca por la originalidad temática y por la forma de gestionar y actualizar sus contenidos y saberes populares.

\section{DISCUSIÓN}

El análisis realizado en las cinco plataformas muestra resultados dispares, y al mismo tiempo cierta similitud entre ellos.

La sociedad actual busca desarrollar las capacidades del ser humano, y en los últimos años se puede detectar que se hace un gran hincapié en las capacidades informacionales, mediáticas, tecnológicas y digitales (Maciá, \& Garreta, 2018). Por ese motivo se observa un abrumador aumento de plataformas digitales para las diferentes etapas educativas.

Como hemos visto, se han analizado 5 plataformas, todas ellas cuentan con una navegabilidad sencilla, aspecto positivo y esencial debido a las edades de los niños y niñas a las que están destinadas, de 3 a 6 años, y las competencias digitales del profesorado, que rozan el suspenso, 2.55 sobre 4 de media, siguiendo algunos estudios realizados recientemente (Fernández et al., 2016).

En lo referente a los aspectos más negativos, hay que resaltar que todas ellas, necesitan mejorar su adaptación a la atención a la diversidad. No se detecta, en general, diversidad cultural, ni racial, cuestión que también reflejan estudios previos realizados con plataformas de materiales didácticos digitales de la etapa de Educación Primaria (Castro-Rodríguez et al., 2017). Este factor interfiere en la accesibilidad de la plataforma y la posibilidad de adaptación de los materiales a los alumnos y alumnas con los que se está trabajando. Añadimos a este aspecto, la necesidad de que los niños y niñas de estas edades se vean reflejados y reflejadas en los materiales que se emplean, que se identifiquen con las actividades, facilitando su aprendizaje y su desarrollo globalizado.

Siguiendo en la dimensión pedagógica, hay que destacar que ninguna de ellas muestra de manera explícita el modelo pedagógico que promueven los materiales ofertados. No obstante, a pesar de que se puede intuir una posición neutral, podríamos decir que la plataforma editorial Bruño se acerca a una enseñanza más tradicional donde el protagonista sigue siendo el docente, mientras que en las demás plataformas dependerá del uso que haga el maestro o maestra en el aula.

En esta etapa educativa se fomenta la autonomía de los más pequeños/as, tal y como se establece en el decreto curricular (Decreto 330/2009), y la imposibilidad de adaptación dificulta esta autonomía. 
En la línea curricular, destaca la falta de interactividad para el/la niño/a en las plataformas. Uno de los objetivos generales de esta etapa es el tratamiento de las tecnologías y su uso y para ello podemos encontrar plataformas como estas. No obstante, ligada al desarrollo de la autonomía, nos encontramos con la capacidad de uso de las plataformas, y en este aspecto las 5 plataformas muestran carencias importantes a la hora de poder manejarlas de manera autónoma por parte del niño.

En este sentido, la plataforma con cierto nivel de interactividad es la plataforma Abalar. No obstante, esta plataforma muestra únicamente el conjunto de recursos que se ofrecen para esta etapa desde la Administración Pública, considerándola más bien un banco de recursos y no tanto una plataforma en sí misma.

Finalmente, es necesario hacer referencia a la plataforma Bruño, en la que denomina materiales digitales a los libros de texto digitalizados al igual que sucedió en investigaciones anteriores (Castro-Rodríguez et al., 2017), tema a debate en la actualidad y que ocupa investigaciones en los últimos años. La plataforma contiene diversos apartados potencialmente útiles para la coordinación docente. No obstante, su adaptabilidad para los niños y niñas es bastante baja.

\section{CONCLUSIONES}

De acuerdo con los resultados obtenidos en esta parte del proyecto de investigación "Los materiales didácticos digitales en la Educación Infantil. Análisis y propuestas para su uso en la escuela y el hogar”, se consideran las siguientes conclusiones:

- Las plataformas analizadas muestran un interesante diseño, destacando en su análisis los aspectos positivos en la dimensión que recibe este mismo nombre.

- El trasfondo de las plataformas es tradicional con una propaganda inicial que lo considera como innovador o diferentes. Engloban materiales mecánicos, individuales y que desarrollan poco la autonomía de los niños y niñas de estas edades.

- Todas ellas están destinadas a docentes y no al alumnado. Además, tienen poca capacidad de adaptarse a las diferentes situaciones o realidades, dejando poco espacio para la modificación por parte del docente.

- Tal y como afirman en Gabarda et al. (2021) y reflejamos en el marco de este artículo, una de las premisas que deben cumplir estas plataformas es la necesidad de que sean colaborativas, en las que los docentes puedan compartir recursos propios. De las 5 plataformas analizadas, ninguna tiene un espacio marcado para ello, sin embargo "EspazoAbalar" y las plataformas libres cuentan con mecanismos para hacerlo.

- En las plataformas comerciales (editoriales), nos encontramos una clasificación estricta de los materiales ofertados, estrictamente relacionado con el currículo, más específicamente con el currículo de etapas superiores, dando a suponer que esta etapa se entiende como algo preparatorio para cursos superiores. Por otro lado, las plataformas libres y de la Administración, clasifican los materiales bajo su propio criterio, o filtros que ayudan al usuario a buscar el material que se necesita.

- En consonancia con el punto anterior, los materiales en las plataformas comerciales se encuentran englobados y terminados, mientras que en las plataformas libres y de la Administración se muestran predominantemente materiales que se pueden combinar entre sí. 
- En el aspecto lingüístico, destaca que tanto las plataformas libres como la Administrativa cuentan con materiales en lengua gallega, mientras que las editoriales hacen una combinación de ambas lenguas, predominando la lengua castellana en la plataforma de Bruño, en la que la lengua gallega está ausente.

- Debido a que en este primer estudio únicamente se han analizado las características pedagógicas de una pequeña muestra de plataformas digitales de materiales didácticos de Educación Infantil, se propone en los siguientes estudios continuar la investigación identificando las visiones de los principales agentes implicados en la utilización de estas plataformas (productores, familias, profesorado y alumnado). Además, en una tercera fase, se realizarán estudios de caso de uso de estas plataformas en aulas y hogares con alumnado de segundo ciclo de Educación Infantil.

En conclusión, dada la sociedad en la que vivimos, las plataformas digitales están dando el salto para adaptarse a las necesidades de nuestra realidad. No obstante, se están quedando en aspectos más superficiales, sin tener en cuenta las necesidades de los niños y niñas a los que van destinados los recursos que se ofertan en ellas. A pesar de ello, se está avanzando en aspectos cuantitativos, encontrándonos, año tras año, con más posibilidades para poder adaptar a las realidades de las aulas de Educación Infantil.

\section{FINANCIACIÓN}

Este artículo es resultado del proyecto de investigación titulado "Los materiales didácticos digitales en la Educación Infantil. Análisis y propuestas para su uso en la escuela y el hogar (RTI2018-093397-B-I00)" financiado por el Fondo Europeo de Desarrollo Regional (FEDER), el Ministerio de Ciencia e Innovación y la Agencia Estatal de Investigación (AEI).

\section{REFERENCIAS}

Altun, S. (2017). The effect of cooperative learning on students achievement and views on the science and technology course. International Electronic Journal of Elementary Education, 7(3), 451-468.

Area, M. (2003). De los web educativos al material educativo web. Comunicación y Pedagogía, 188, 32-38.

Area, M. (2019). Guía para la producción y uso de materiales didácticos digitales: recomendaciones de buenas prácticas para productores, profesorado y familias. http://edullab.webs.ull. es/wordpress/guia-bbpp-mdd/

Castro-Rodríguez, M.M., De Castro, A., \& Hernández, V.M. (2017). Análisis de plataformas educativas digitales comerciales españolas destinadas a Educación Primaria. Revista Latinoamericana de Tecnología Educativa, 16(2), 49-62. https://doi. org/10.17398/1695-288X.16.2.49
Chaudron, S., Di Giogia, R., \& Gemo, M. (2018). Young children (‘8) and digital technology, a qualitative study across Europe. JRC Science for Policy Report https://doi.org/10.2760/294383

Decreto 330/2009, de 4 de junio, por el que se establece el currículo de la educación infantil en la Comunidad Autónoma de Galicia. Xunta de Galicia. Diario Oficial de Galicia, 121, de 23 de junio de 2009, pp. 10773-10799. https://politicasocial. xunta.gal/sites/w polso/files/arquivos/normativa/decreto 3302009 cas.pdf

Fernández, J.C., Fernández, M.C., \& Cebreiro, B. (2016). Competencias en TIC del profesorado en Galicia: variables que inciden en las necesidades formativas. Innovación Educativa, (26), 215-231.

Flórez, M., Aguilar, A.J., Hernández, Y.K., Salazar, J.P., Pinillos, J.A., \& Pérez, C.A. (2017). Sociedad del conocimiento, las TIC 
y su influencia en la educación. Revista Espacios, 38(35), e39. http://hdl.handle.net/20.500.12442/1770

Gabarda, V., Rodríguez, N., \& González, C. (2021). Los materiales didácticos digitales en educación infantil: análisis de repositorios institucionales. Revista Iberoamericana de Educación, 85(1), 61-79. https://doi.org/10.35362/rie8514069

Gonçalves, D. (2015). Competências profissionais e desenvolvimento docente. En J. Machado \& J. Matias (Eds.), Professores, escola e município - formar, conhecer e desenvolver (pp. 4252). UCP.

Gonzales, A. (2016). The contemporary US digital divide: From initial access to technology maintenance. Information, Communication \& Society, 19(2), 234-248. https://doi. org/10.1080/136911

González, C.J., Martín, S., \& Vega, A. (2018). Portales educativos: la producción de materiales didácticos digitales. @tic Revista d'innovació educativa, (20), 89-97. https://doi.org/10.7203/ attic.20.12139

Gottschalk, F. (2019). Impacts of technology use on children: Exploring literature on the brain, cognition and well-being. In OECD Education Working Papers. OECD Publishing. https:// dx.doi.org/10.1787/8296464e-en

Gutiérrez, F. (2018). Sobre la nueva ecología de medios en la era digital: hacia un nuevo inventario de efectos. Razón y Palabra, 22(1_100), 237-254.

Hernández, R.M. (2017). Impacto de las TIC en la educación: Retos y Perspectivas. Propósitos y representaciones, 5(1), 325-347. http://dx.doi.org/10.20511/pyr2017.v5n1.149

Hooft, J. (2018). New technologies and $21^{\text {st }}$ century children: Recent trends and outcomes. In OECD Education Working Papers. OECD Publishing. https://dx.doi.org/10.1787/e071a505-en

Maciá, M., \& Garreta, J. (2018). Accesibilidad y alfabetización digital: barreras para la integración de las TIC en la comunidad familia/escuela. Revista de Investigación Educativa, 36 (1), 239- 257. https://doi.org/10.6018/rie.36.1.290111

Pérez, R., Mercado, P., Martínez, M., Mena, E., \& Partida, J.A. (2018). La sociedad del conocimiento y la sociedad de la información como la piedra angular en la innovación tecno- lógica educativa. RIDE. Revista Iberoamericana para la Investigación y el Desarrollo Educativo, 8(16), 847-870.https://doi. org/10.23912/ride.v8i16.371

Rodríguez, J., \& Area, M. (2019). Resumen Memoria Técnica del Proyecto Infanci@ Digit@l (RTI2018093397-B-100). Los materiales didácticos digitales en la Educación Infantil. Análisis y propuestas para su uso en la escuela y en el hogar. Documento oficial, difusión restringida.

Sosa, M.J., \& Valverde, J.V. (2020). Perfiles docentes en el contexto de la transformación digital de la escuela. Bordón. Revista de Pedagogía, 72(1), 151-173. https://doi.org/10.13042/Bordon. 2020.72965 